\title{
Pantoprazole Decreases Cell Viability and Function of Human Osteoclasts In Vitro
}

\author{
Markus Prause, Claudine Seeliger, Marina Unger, Elizabeth Rosado Balmayor, \\ Martijn van Griensven, and Alexander Tobias Haug
} Experimental Trauma Surgery, Department of Trauma Surgery, Klinikum Rechts der Isar, Technical University Munich,
Ismaninger Straße 22, 81675 Munich, Germany

Correspondence should be addressed to Markus Prause; prause@uchir.me.tum.de

Received 28 August 2014; Revised 7 November 2014; Accepted 12 November 2014

Academic Editor: Frank Hildebrand

Copyright (C) 2015 Markus Prause et al. This is an open access article distributed under the Creative Commons Attribution License, which permits unrestricted use, distribution, and reproduction in any medium, provided the original work is properly cited.

\begin{abstract}
Proton pump inhibitors (PPIs) are commonly prescribed drugs that decrease stomach acidity and are thus often used to treat gastroesophageal reflux disease and as a preventative agent for the adverse effects of nonsteroidal anti-inflammatory drugs on the stomach mucosa. In recently published literature, an association between proton pump inhibitor administration and increased fracture risk has been stated. In order to reveal the underlying pathomechanisms of these observations, the effects of pantoprazole, a representative of the proton pump inhibitors, on human osteoclasts in vitro were evaluated in this study. Osteoclasts were stimulated with increasing concentrations of pantoprazole ranging from $0 \mu \mathrm{g} / \mathrm{mL}$ to $10 \mu \mathrm{g} / \mathrm{mL}$ over a period of seven days. Cell viability and tartrate-resistant acid phosphatase (TRAP) activity assays were performed after 1 day, 3 days, and 7 days, respectively. Here, stimulated osteoclasts presented a significantly lower viability and TRAP activity than the negative controls. Osteoclast-specific gene expression was evaluated after seven days and revealed no significant differences between all samples. Overall, the bone degrading and resorptive function of osteoclasts is inhibited by the administration of proton pump inhibitors. While PPI-related fractures through "basic multicellular unit" dysfunction are unlikely, the underlying pathomechanism remains unknown.
\end{abstract}

\section{Introduction}

Pantoprazole is one of the most frequently used proton pump inhibitors (PPIs) in the clinical setting. By irreversibly inhibiting $\mathrm{H}+/ \mathrm{K}+\mathrm{ATPase}$, or proton pump, in gastric cells, it strongly reduces the proton influx into the gastric lumen and therefore the effective stomach acidity. It is therefore used in the treatment of gastroesophageal reflux disease, in eradication treatment of Helicobacter pylori infection, and in combination with nonsteroidal anti-inflammatory drugs (NSAIDs) for prophylaxis of stress ulcers [1-3]. Although the administration of PPIs in a wide range of medical disciplines is necessary, they are often wrongly administered chronically and without a clear indication [4].

The administration of various PPIs has been shown to be significantly associated with an increased fracture risk, while a direct causal link between the two is not yet proven [57]. While short-term administration of PPIs already poses a moderately increased risk, long-term administration over a year or more seems to further increase the fracture risk $[8,9]$.
Insogna hypothesized a cell-independent mechanism why bone density should decrease under PPI therapy. According to his study, altered gastric acidity may lead to reduced calcium absorption, which would ultimately increase the rate of bone loss [10]. Similarly, Vestergaard et al. hypothesized a secondary hyperparathyroidism and subsequent osteoporosis as a possible cause for the increased fracture risk [11].

Our study group has previously investigated the influence of pantoprazole on osteoblasts in vitro. Osteoblasts represent a subpopulation of bone cells that synthesize the bone matrix. Pantoprazole seems to have beneficial effects on the viability of osteoblasts and their ability to synthesize new bone matrix [12]. The functional counterpart to osteoblasts within the basic multicellular units is osteoclasts, which actively degrade and resorb bone matrix. Together, these cellular units form a functional entity that secures dynamic bone homeostasis in order to meet the requirements of constant load transmission. It has been described that, additionally to their inhibitory effects on gastric proton pumps, PPIs may inhibit a proton 
pump in osteoclasts also known as the vacuolar ATPase [13]. It is mainly responsible for creating an acidic environment between the ruffled border of osteoclasts and bone tissue. In this acidic environment at the bone-apposed plasma membrane of the osteoclast, lytic enzymes are activated and bone is resorbed within the process of remodeling [14]. VATPases are related yet not identical to the proton pump that is located in parietal cells. Consequently, effects of PPIs on VATPases are less apparent than on gastric proton pumps [15].

Since no pathomechanism has yet been found that adequately explains or indeed proves a causal correlation between the use of PPIs and an increased fracture risk, the aim of this study was to investigate the effect of an important representative of the proton pump inhibitorspantoprazole-on a cellular level.

\section{Materials and Methods}

The plastic labware used in our experiments was acquired from Sarstedt (Nümbrecht, Germany). The culture plates were acquired from GE Healthcare (Little Chalfont, UK). The chemicals were acquired from Sigma-Aldrich (St. Louis, MO, USA). Dentine chips were kindly provided by Associate Professor Susanne Mayer, MD, Laboratory for Biomechanics and Experimental Orthopaedics, Department of Orthopaedics, Ludwig-Maximilians-University, Munich, Germany.

2.1. Isolation of Peripheral Blood Mononuclear Cells (PBMCs). Buffy coats (i.e., portions of donated blood that contain a concentrated amount of leucocytes) of male and female donors $(N=4)$ ranging from 50 to 67 years in age were obtained from Bayerisches Rotes Kreuz Blutspendedienst (Bavarian Red Cross Blood Donation Centre, Ulm, Germany). We selected donors of higher age since the prescription and therefore potential risks of PPIs are more imminent in older than in younger patients. After resuspension with $250 \mathrm{~mL}$ Dulbecco's phosphate buffered saline (DPBS) per buffy coat, $30 \mathrm{~mL}$ of suspension was layered on top of $20 \mathrm{~mL}$ lymphocyte separation medium (Biowest, Nuaillé, France) with a density of $1077 \mathrm{~kg} / \mathrm{m}^{3}$. After 20 minutes of centrifugation at $1000 \mathrm{~g}$ without brakes, the PBMCs were isolated from the formed interphase. Afterwards, the cells were resuspended in DPBS and centrifuged again at $650 \mathrm{~g}$ for 10 minutes. This last step was repeated once more for purification. Finally, the PBMCs were resuspended in cultivation medium which consisted of $500 \mathrm{~mL}$ alpha modification minimum essential medium (PAA Laboratories GmbH, Cölbe, Germany), 10\% fetal calf serum (FCS), $100 \mathrm{U} / \mathrm{mL}$ penicillin, and $10 \mu \mathrm{g} / \mathrm{mL}$ streptomycin. The cells were then plated at a concentration of 3 million cells per well on 48-well plates for cell experiments and 6 million cells per well on 24-well plates for gene expression analysis.

2.2. Osteoclast Differentiation. 24 hours after isolation, the isolation medium was exchanged for cultivation medium that contained $25 \mathrm{ng} / \mathrm{mL}$ macrophage colony-stimulating factor (M-CSF). All following media exchanges were performed on Mondays, Wednesdays, and Fridays. After a week of M-CSF stimulation, cultivation medium containing $12.5 \mathrm{ng} / \mathrm{mL} \mathrm{M}-\mathrm{CSF}$ and $10 \mathrm{ng} / \mathrm{mL}$ receptor activator of nuclear factor $\kappa \mathrm{B}$ (RANKL) was administered. For the following two weeks, the monocyte cultures were stimulated with medium containing $20 \mathrm{ng} / \mathrm{mL}$ RANKL.

2.3. Experimental Setup. After 24 days of differentiation, the osteoclasts were stimulated every other day with freshly prepared pantoprazole (PP) at the following concentrations: $0 \mu \mathrm{g} / \mathrm{mL}$ (negative control), $0.1 \mu \mathrm{g} / \mathrm{mL}, 1 \mu \mathrm{g} / \mathrm{mL}, 3 \mu \mathrm{g} / \mathrm{mL}$, and $10 \mu \mathrm{g} / \mathrm{mL}$. These include the concentrations that correspond to the in vivo serum concentrations $(1 \mu \mathrm{g} / \mathrm{mL}$ and $3 \mu \mathrm{g} / \mathrm{mL})$ of the two most common prescription doses of pantoprazole (20 $\mathrm{mg}$ and $40 \mathrm{mg}$ ). This was done to reveal effects that are most relevant to clinical application. The effects of pantoprazole on osteoclast function, metabolism, and gene expression were analysed on days 1,3, and 7 after the initial stimulation.

2.4. Osteoclast Phenotype: von Kossa Staining. In order to demonstrate and prove the osteoclastic phenotype and the ability to absorb mineralised matrix, PBMCs were plated on a culture plate coated with a thin layer of calcium phosphate (Corning, Tewksbury, MA, USA). After differentiation and stimulation, the medium was removed and the osteoclasts were incubated with $\mathrm{NaClO}$ for 5 minutes at room temperature. Afterwards, the wells were washed twice with $\mathrm{dH}_{2} \mathrm{O}$ and subsequently incubated with a $3 \%$ solution of silver nitrate in $\mathrm{H}_{2} \mathrm{O}$ for 30 minutes at room temperature. The silver nitrate solution was removed and the wells were washed repeatedly in $\mathrm{dH}_{2} \mathrm{O}$ over 10 minutes. Then, a $1 \%$ solution of pyrogallol in $\mathrm{H}_{2} \mathrm{O}$ was added and incubated for 3 minutes. After two additional washing steps with $\mathrm{dH}_{2} \mathrm{O}$, the samples were fixated with a $5 \%$ solution of sodium thiosulfate in $\mathrm{H}_{2} \mathrm{O}$ for 5 minutes. After multiple washing steps with $\mathrm{dH}_{2} \mathrm{O}$, pictures were taken using the BZ9000 fluorescence microscope (Keyence, Osaka, OSK, Japan). Through the von Kossa staining, the remaining calcium phosphate appears as a dark grey and the resorption pits appear as a light grey to white shade.

2.5. Osteoclast Phenotype: Toluidine Blue Staining. Toluidine blue staining was performed on osteoclasts that were cultivated on dentine chips in suspension culture plates to show the formation of resorption lacunae. The cells were lysed with sodium hypochlorite solution and wiped off the dentine chips with a paper towel. After short immersion in $1 \%$ toluidine blue solution, pictures were taken with the BZ9000 fluorescence microscope (Keyence, Osaka, OSK, Japan).

2.6. Osteoclast Phenotype: TRAP Staining. To visualise the presence and resorptive activity of osteoclasts and thus prove the successful differentiation, TRAP staining was performed on control samples after 1 day, 3 days, and 7 days. The cells were fixated with a solution of $10 \%$ DPBS, $4 \%$ formaldehyde, and $0.2 \%$ Triton $\mathrm{X}-100$ in $\mathrm{dH}_{2} \mathrm{O}$ for 5 minutes. The staining buffer consisted of $40 \mathrm{mM}$ sodium acetate and $10 \mathrm{mM}$ disodium tartrate dihydrate in $\mathrm{ddH}_{2} \mathrm{O}(\mathrm{pH}=5) .0 .01 \%$ naphthol 
TABLE 1: Primers used for RT-PCR analysis.

\begin{tabular}{lrc}
\hline Gene & Forward primer $\left(5^{\prime}-3^{\prime}\right)$ & Reverse primer $\left(5^{\prime}-3^{\prime}\right)$ \\
\hline$A C T B$ & ACAGAGCCTCGCCTTTGCCGAT & GCGAAGCCGGCCTTGCACAT \\
NFאB $B$ GCGCCGCTTAGGAGGGAG & GAAGGTATGGGCCATCTGCTGTT \\
TRAP & CCCTCGGAGAAACTGCATCAT & CATGTCCATCCAGGGGGAGA \\
VATP & GGGCTGCTCATGTTCCTCTT & CACGTGGTTGAAGACTCCGA \\
NFATC1 & GCTTTAAAAAGGCAGGAGGCA & GAGGAAAGTCATCGAGGGGC \\
\hline
\end{tabular}

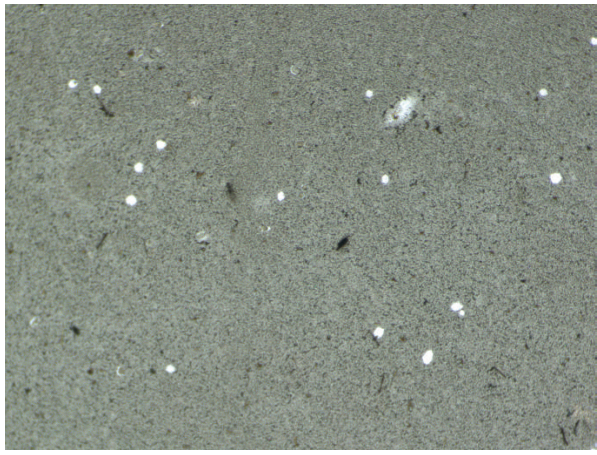

(a)

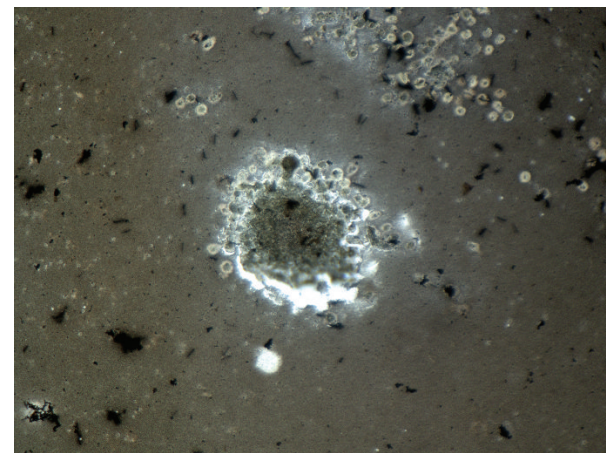

(b)

FIGURE 1: Depicted are two exemplary fields of view of the von Kossa-stained calcium phosphate plate. The pictures were taken of samples that were not stimulated with pantoprazole, 7 days after differentiation. Multiple resorption zones (white spots) where osteoclasts were located prior to lysis are visible at 20x magnification (a). A large cluster of osteoclasts is visible in the middle of the picture with several smaller clusters in the periphery of the picture at 10x magnification (b).

AS-MX phosphate, $0.06 \%$ Fast Red Violet LB salt, and $1 \% \mathrm{~N}$ $\mathrm{N}$ dimethylformamide were added to get the final staining solution. After the fixation, the cells were air-dried at room temperature. Finally, the staining solution was added and incubated for 20 minutes at $37^{\circ} \mathrm{C}$. After a washing step with DPBS, pictures were taken using the BZ-9000 fluorescence microscope (Keyence, Osaka, OSK, Japan).

2.7. TRAP Activity. In order to determine the level of osteoclast resorption activity, the TRAP activity of the osteoclasts was assessed. The assay buffer consisted of $100 \mathrm{mM}$ sodium acetate and $50 \mathrm{mM}$ disodium tartrate dihydrate in $\mathrm{ddH}_{2} \mathrm{O}$ ( $\mathrm{pH}$ 5.5). The substrate buffer solution consisted of $5 \mathrm{mM} 4$ nitrophenyl phosphate disodium salt hexahydrate in assay buffer. For TRAP activity analysis, $50 \mu \mathrm{L}$ of cell supernatant of each sample was transferred to a 96-well plate. The aliquots were incubated at $37^{\circ} \mathrm{C}$ with $150 \mu \mathrm{g} / \mathrm{mL}$ assay buffer per well for 1 hour. A standard curve was prepared by stepwise dilution of $1 \mathrm{mM}$ 4-nitrophenol in a 1:4 solution of osteoclast culture medium and assay buffer. After the total incubation time, the reaction was stopped using $50 \mu \mathrm{L}$ of $3 \mathrm{M} \mathrm{NaOH}$ solution. Finally, the absorbance was detected at $\lambda=405 \mathrm{~nm}$. TRAP activity was calculated according to the standard curve.

2.8.3-(4,5-Dimethylthiazol-2-yl)-2,5-diphenyltetrazolium Bromide (MTT) Assay. In order to quantify cell viability, the MTT assay was performed and set in relation to the negative control. The samples were incubated with $1.2 \mathrm{mM}$ thiazolyl blue solution for 120 minutes at $37^{\circ} \mathrm{C}$ and $5 \% \mathrm{CO}_{2}$. Afterwards, the supernatant was removed and the remaining formazan crystals were resolved in a dimethyl sulfoxide solution containing $10 \%$ sodium dodecyl sulfate and $0.6 \%$ acetic acid. The formazan content was determined through photometrical measurements at $\lambda=570 \mathrm{~nm}$ and $\lambda=690$ nm.

2.9. Gene Expression. In order to evaluate gene expression activity, semiquantitative analyses of reverse transcription polymerase chain reaction (RT-PCR) products were performed. The total RNA was isolated from the cells using TriFast (Sigma-Aldrich, St. Louis, MO, United States), phenol chloroform extraction, and subsequent ethanol precipitation. The RNA was transcribed into complementary DNA (cDNA) using the First-Strand cDNA Synthesis Kit (Fermentas, St. Leon-Rot, Germany). RT-PCRs were performed after a standard protocol. The respective primers of the investigated genes are listed in Table 1. Ethidium bromide was used for visualisation of the PCR products that were separated in $1.8 \% \mathrm{w} / \mathrm{v}$ agarose gels. The densitometric analysis of the generated signals was performed with the ImageJ software (NIH, Bethesda, MD, USA). The housekeeping gene $\beta$-actin $(A C T B)$ was used for normalisation.

\section{Statistics}

The experiments were performed independently on four individual specimens $(N=4)$. The measurements were performed as duplicates or more $(n \geq 2)$. The gathered results are depicted as mean \pm SEM. Kruskal-Wallis test and Dunn's multiple comparisons test (GraphPad Prism Software, El Camino Real, USA) were used for data comparison. The minimal value of significance was $P<0.05$. 


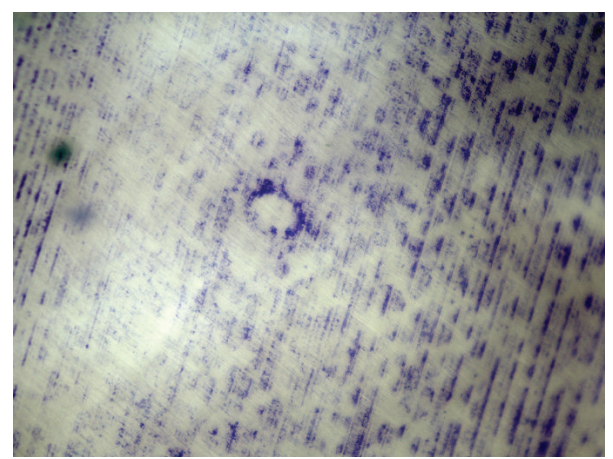

FIgURE 2: A resorption lacuna is made visible on the dentine chip through toluidine blue staining. The picture was taken under light microscopy at 10x magnification, 7 days after differentiation. The sample in this picture consisted of osteoclasts that were not stimulated with pantoprazole.

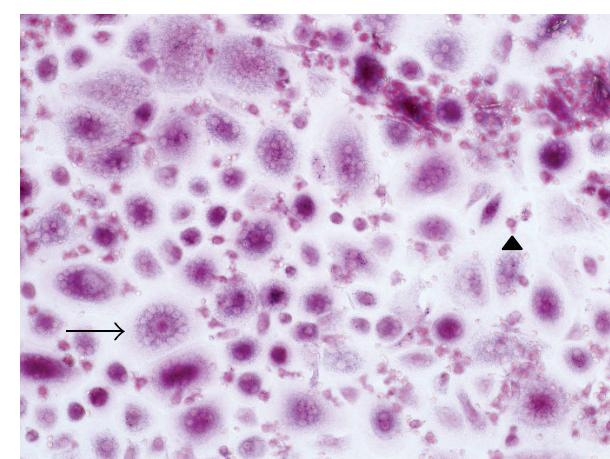

(a)

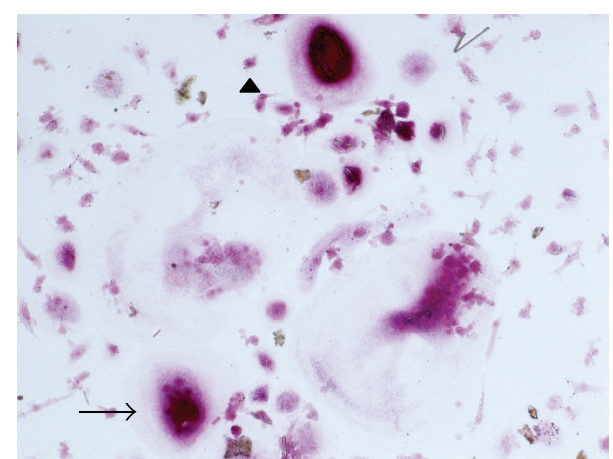

(b)

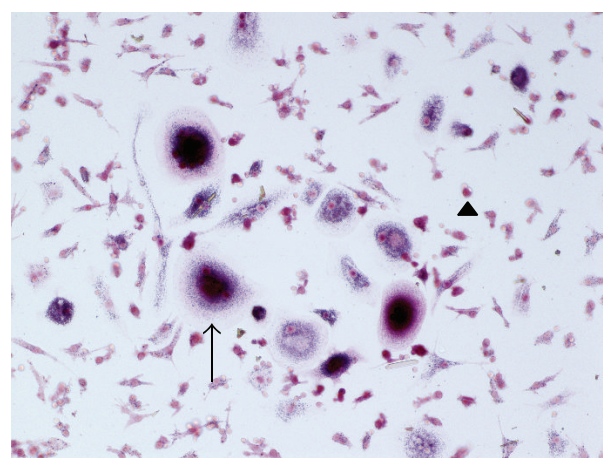

(c)

FiguRE 3: Depicted are three representative fields of view of TRAP-stained samples, 1 day (a), 3 days (b), and 7 (c) days after differentiation and without pantoprazole stimulation. Visible are multinucleated osteoclasts (arrows) as well as mononuclear cells that have not yet merged (arrowheads).

\section{Results}

4.1. von Kossa Staining/Toluidine Blue Staining/TRAP Staining. The stainings were performed on negative control samples of each donor 7 days after completed differentiation. The von Kossa-stained samples show that the differentiated cells actively resorbed calcium phosphate (Figures 1(a) and 1(b)) while the exemplary toluidine blue-stained dentine chip shows the formation of Howship's lacuna (Figure 2). Furthermore, the strong accumulation of TRAP stain in the depicted cells suggests successful differentiation of the PBMCs into active osteoclasts after days 1, 3, and 7 after differentiation (Figures 3(a), 3(b), and 3(c)).
4.2. TRAP Activity. TRAP activity assessed on days 1 and 3 after initial differentiation showed no significant differences between the control and samples stimulated with PP (Figures 4(a) and 4(b)). On day 7, however, the TRAP activity of the $0.1 \mu \mathrm{g} / \mathrm{mL}$ and $1 \mu \mathrm{g} / \mathrm{mL}$ PP groups showed a decrease of $6.12 \%$ $(P<0.05)$ and $8.56 \%(P<0.01)$, respectively (Figure $4(\mathrm{c}))$.

4.3. MTT Assay. The performed MTT assay revealed a decreased viability in osteoclast cultures stimulated with PP. On day 1 , the viability of the samples exposed to $3 \mu \mathrm{g} / \mathrm{mL}$ $\mathrm{PP}$ was decreased by $13.04 \%(P<0.05)$. The viability of the other sample groups decreased as well, yet without 


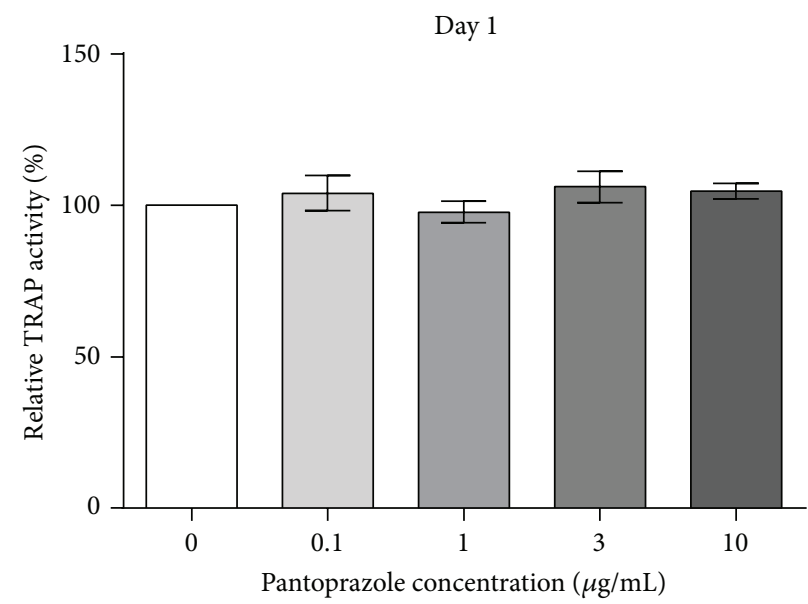

(a)

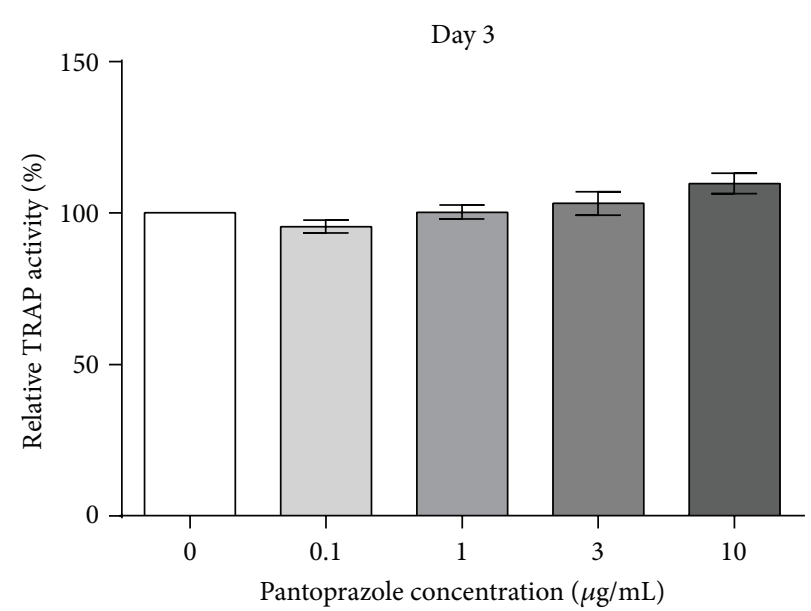

(b)

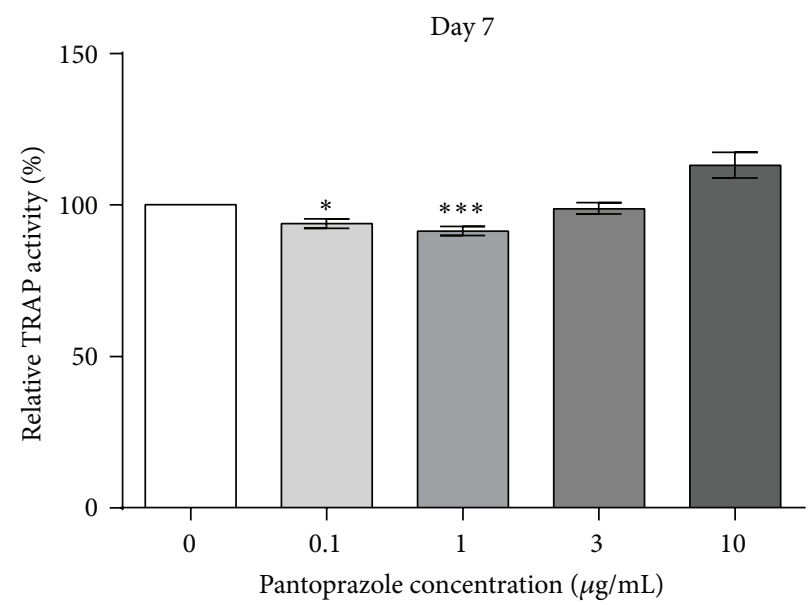

(c)

FIgURE 4: The relative TRAP activity of osteoclast samples $(N=4, n=6)$, that is, the activity of samples stimulated with pantoprazole normalized to untreated samples, was assessed on days 1,3 , and 7 of stimulation. The results were accordingly expressed as a percentage of the negative control. Shown are column bar graphs of means \pm SEM. ${ }^{*} P<0.05$ and ${ }^{* * *} P<0.001$ versus the untreated controls.

statistical significance (Figure 5(a)). On day 3, stimulation with $1 \mu \mathrm{g} / \mathrm{mL}$ PP or more led to a decreased viability and was also statistically insignificant (Figure 5(b)). On day 7, the viability of samples that were stimulated with $10 \mu \mathrm{g} / \mathrm{mL}$ PP was decreased by $15.33 \%(P<0.01)$ (Figure 5(c)).

4.4. Gene Expression. The gene expression analysis of nuclear factor kappa-light-chain-enhancer of activated B cells $(N F \kappa B)$, tartrate-resistant acid phosphatase (TRAP), a subunit of the V-ATPase (VATP), and nuclear factor of activated T-cells, cytoplasmic 1 (NFATc1), revealed no significant differences after 7 days of stimulation (Figures 6(a), 6(b), 6(c), and 6(d)).

\section{Discussion}

The question whether PPIs have detrimental effects on bone metabolism and may in fact cause an increased fracture risk, as well as being a risk factor for other pathologies, such as community-acquired pneumonia, is of great interest in the clinical setting $[5,6,8,16,17]$. The fact that most standard pain management therapies include the concomitant administration of PPIs, in many cases over long periods of time, would make negative effects particularly harmful. In this study, we explored the effects of pantoprazole, one commonly prescribed PPI, on a cellular level.

After successful initial differentiation of the isolated PBMCs into osteoclasts, our stimulation experiments were carried out over a period of 7 days. The performed cell viability assays, as well as the TRAP activity assays, suggest that human osteoclasts are significantly inhibited in their overall ability to degrade and absorb bone matrix. This is in accordance with the findings of Sheraly et al., who showed in their in vivo experiments that pantoprazole $(0.5 \mathrm{mg} / \mathrm{mL})$ and omeprazole $(40 \mathrm{mg} / \mathrm{mL})$ decrease the resorptive activity of osteoclasts when instilled into calcium phosphate cement [18]. Additionally, Hyun et al., who investigated the effects of omeprazole on murine osteoclast-like RAW 264.7 cells, revealed that osteoclast function is overall inhibited, while osteoblast function is enhanced, leading to 


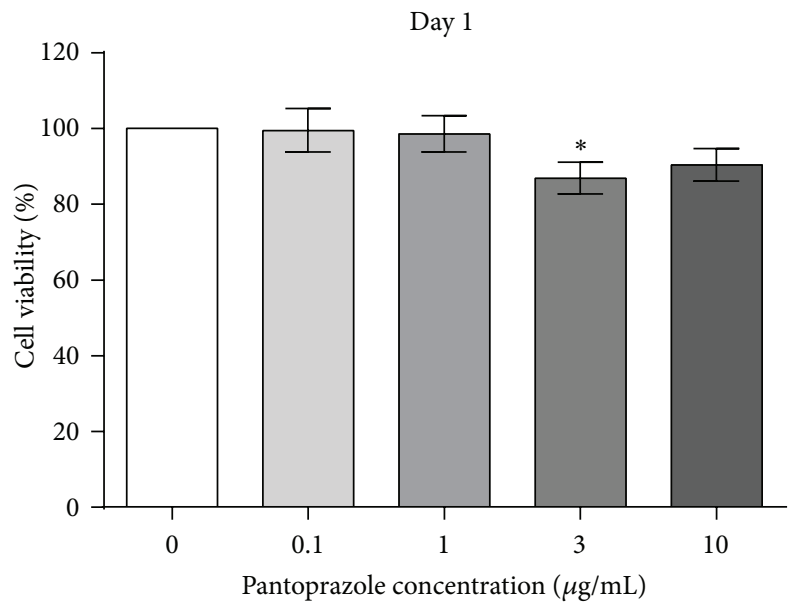

(a)

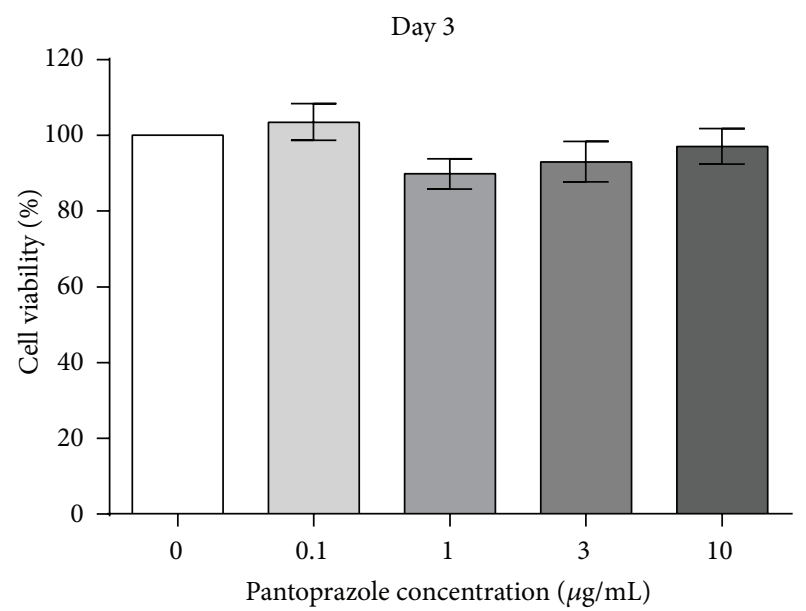

(b)

Day 7

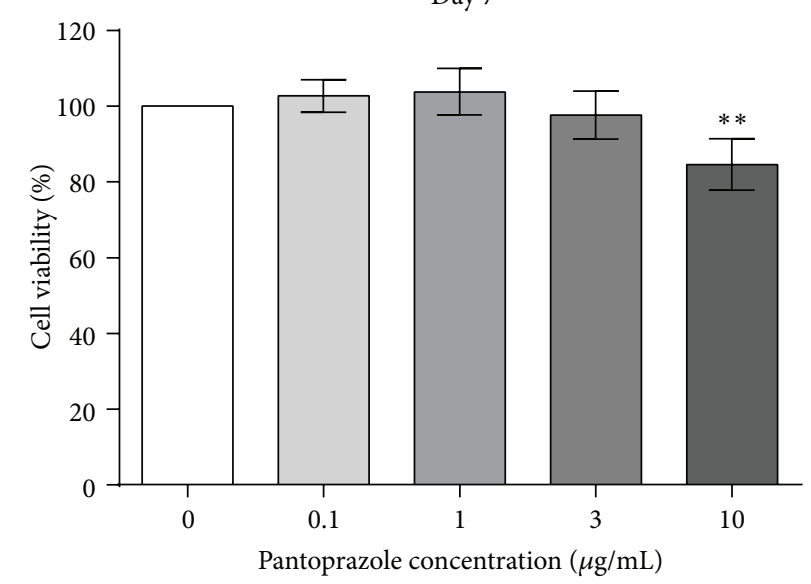

(c)

FIGURE 5: The MTT cell viability assays performed on days 1,3 , and 7 of stimulation with pantoprazole $(N=4, n=6)$. The viability in relation to the respective negative control was assessed. The results were expressed as percentages of the negative control values. Shown are column bar graphs of means \pm SEM. ${ }^{*} P<0.05$ and ${ }^{* *} P<0.01$ versus the untreated controls.

an osteopetroricket-like bone matrix [19]. In synopsis of our studies on human osteoblasts and osteoclasts, as well as various studies that confirm the inhibitory effects of PPI on osteoclast function, the development of osteoporosis through effects on a cellular level as a causal link between PPI treatment and an increased fracture risk seems unlikely $[6,12$, 20]. Accordingly, Targownik et al., who used a multivariate linear regression model on a set of data from the Canadian Multicentre Osteoporosis Study to determine whether there is a relevant decrease in bone mineral density (BMD) after administration of PPIs, revealed that there was no association between PPI usage and accelerated BMD loss over a ten-year period, although PPI users had a lower average baseline BMD [21].

However, not all studies agree on the effects of PPIs on bone homeostasis. In an early study by Mizunashi et al., for example, the administration of omeprazole led to an increase in serum levels of parathyroid hormone and subsequently TRAP, alkaline phosphatase, and bone glia protein (osteocalcin) while decreasing urinary excretion of calcium. This was thought to suggest overall increased bone turnover
[22]. Costa-Rodrigues et al. on the other hand proposed in their study that there is the possibility of a dose-dependent decrease in bone turnover with the administration of PPIs [23]. Finally, several meta-analyses that were performed on recent observational studies hypothesized that the heterogeneity of results concerning the increased fracture risk could be due to hitherto unknown confounders and called for further investigation $[6,7]$.

\section{Conclusions}

PPIs are commonly used in combination with NSAID in pain management regiments for patients with bone-related pathologies. An increased fracture risk implying adverse effects on bone homeostasis would especially limit the safe application of this group of drugs as first line therapy in this context. In synopsis of this and several other in vitro studies, we think that the development of osteoporosis through direct cellular impairment is unlikely. Under PPI stimulation, the ability of osteoclasts to resorb mineralized matrix seems to 


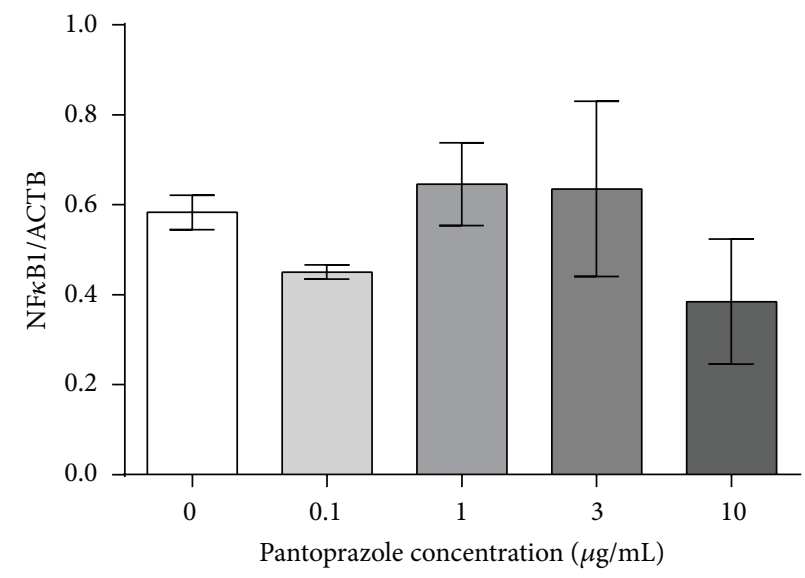

(a)

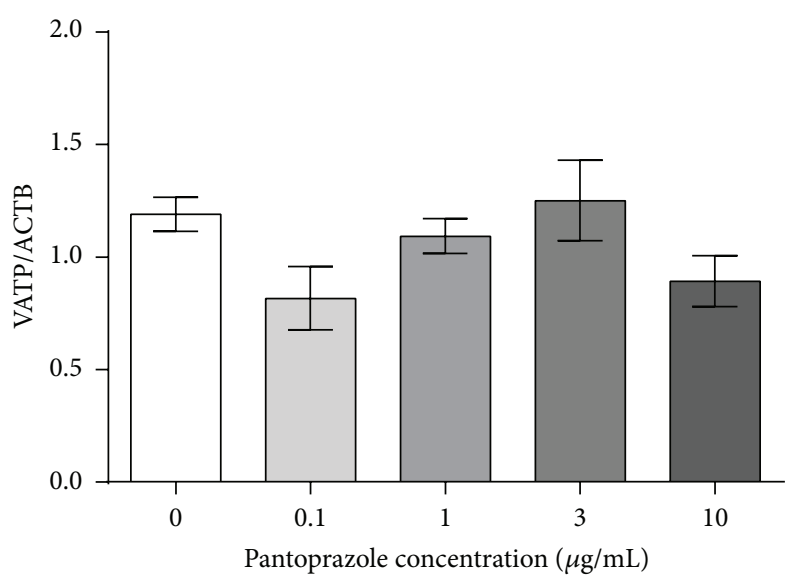

(c)

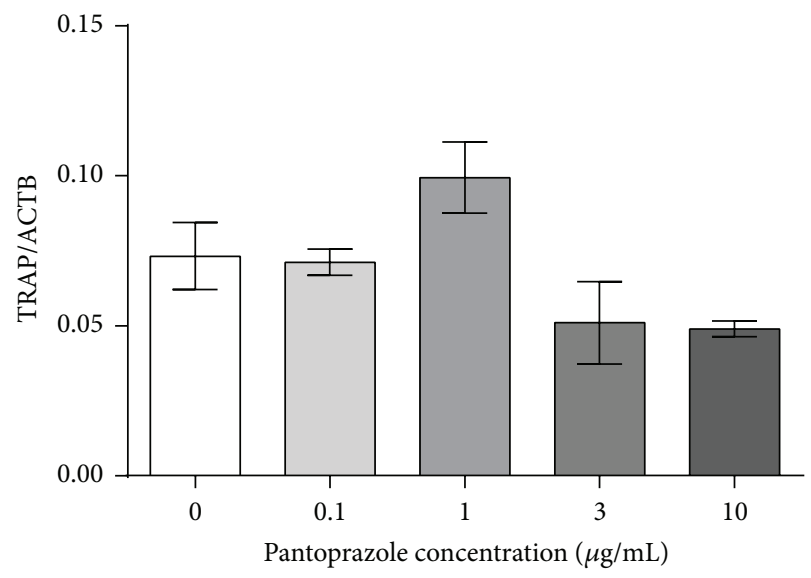

(b)

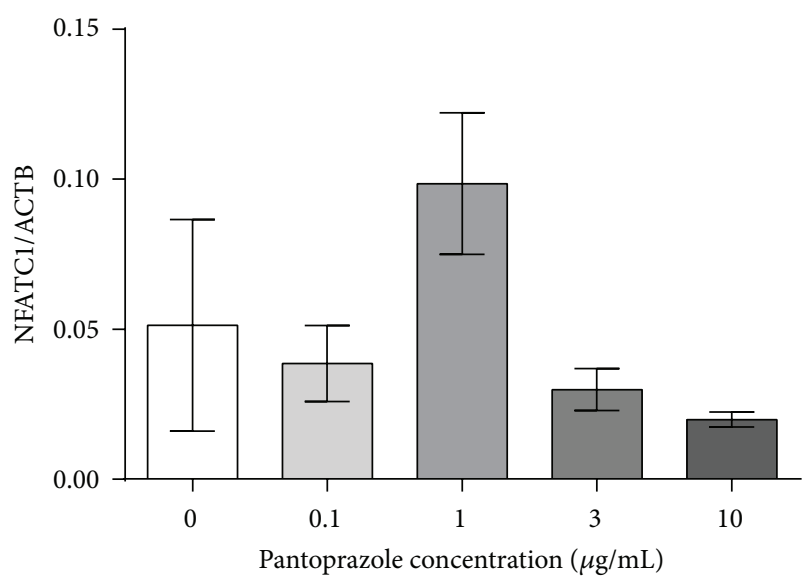

(d)

Figure 6: Gene expression analysis of $N F \kappa B$ (a), TRAP (b), VATP (c), and NFATC1 (d) was performed after 7 days of stimulation with pantoprazole $(N=4, n=2)$. The results were normalized to the housekeeping gene $\beta$-actin (ACTB). Shown are column bar graphs of means \pm SEM.

be inhibited, while the ability of osteoblasts to synthesize new matrix is increased.

Reduced bone turnover as a result of the decreased resorption rate could potentially contribute to an increased fracture risk, as the bone matrix is not optimally adapted to its constantly changing load. Additionally, cell-independent causes of osteoporosis, for example, decreased calcium absorption or increased parathyroid hormone excretion, remain possible explanations for the association between PPI use and fracture risk. Finally, it must also be considered that the increased fracture risk found in observational studies is not causally related to the administration of PPIs but rather caused by an unknown confounder and therefore factually nonexistent. To prove a causal link between the use of PPIs and an increased fracture risk, further investigations into potential confounding factors as well as in vivo studies to further illustrate effects of the drugs on the cells in their natural environment are necessary.

\section{Conflict of Interests}

The authors declare that there is no conflict of interests regarding the publication of this paper.

\section{Acknowledgments}

The authors would like to thank Fritz Seidl, M.A. Interpreting and Translating, for the linguistic revision and improvement of this paper. They thank Professor Ignatius, DVM, Institute for Orthopaedic Research and Biomechanics, Ulm, for providing the protocol for the von Kossa osteoclast phenotype determination.

\section{References}

[1] P. O. Katz, L. B. Gerson, and M. F. Vela, "Guidelines for the diagnosis and management of gastroesophageal reflux disease," The American Journal of Gastroenterology, vol. 108, no. 3, pp. 308-328, 2013.

[2] J. L. Wallace, "Prostaglandins, NSAIDs, and gastric mucosal protection: why doesn't the stomach digest itself?" Physiological Reviews, vol. 88, no. 4, pp. 1547-1565, 2008.

[3] W. Alhazzani, F. Alenezi, R. Z. Jaeschke, P. Moayyedi, and D. J. Cook, "Proton pump inhibitors versus histamine 2 receptor antagonists for stress ulcer prophylaxis in critically ill patients: a systematic review and meta-analysis," Critical Care Medicine, vol. 41, no. 3, pp. 693-705, 2013. 
[4] S. Chubineh and J. Birk, "Proton pump inhibitors: the good, the bad, and the unwanted," Southern Medical Journal, vol. 105, no. 11, pp. 613-618, 2012.

[5] X. Ye, H. Liu, C. Wu et al., "Proton pump inhibitors therapy and risk of hip fracture: a systematic review and meta-analysis," European Journal of Gastroenterology \& Hepatology, vol. 23, no. 9, pp. 794-800, 2011.

[6] E. W. Yu, T. Blackwell, K. E. Ensrud et al., "Acid-suppressive medications and risk of bone loss and fracture in older adults," Calcified Tissue International, vol. 83, no. 4, pp. 251-259, 2008.

[7] S. Ngamruengphong, G. I. Leontiadis, S. Radhi, A. Dentino, and K. Nugent, "Proton pump inhibitors and risk of fracture: a systematic review and meta-analysis of observational studies," The American Journal of Gastroenterology, vol. 106, no. 7, pp. 1209-1218, 2011.

[8] L. E. Targownik and W. D. Leslie, "The relationship among proton pump inhibitors, bone disease and fracture," Expert Opinion on Drug Safety, vol. 10, no. 6, pp. 901-912, 2011.

[9] Y.-X. Yang, J. D. Lewis, S. Epstein, and D. C. Metz, "Long-term proton pump inhibitor therapy and risk of hip fracture," The Journal of the American Medical Association, vol. 296, no. 24, pp. 2947-2953, 2006.

[10] K. L. Insogna, "The effect of proton pump-inhibiting drugs on mineral metabolism," The American Journal of Gastroenterology, vol. 104, supplement 2, pp. S2-S4, 2009.

[11] P. Vestergaard, L. Rejnmark, and L. Mosekilde, "Proton pump inhibitors, histamine $\mathrm{H}_{2}$ receptor antagonists, and other antacid medications and the risk of fracture," Calcified Tissue International, vol. 79, no. 2, pp. 76-83, 2006.

[12] M. Prause, C. Seeliger, M. Unger, M. van Griensven, and A. T. Haug, "Pantoprazole increases cell viability and function of primary human osteoblasts in vitro," Injury, vol. 45, no. 8, pp. 1156-1164, 2014.

[13] J. Tuukkanen and H. K. Väänänen, "Omeprazole, a specific inhibitor of $\mathrm{H}^{+}-\mathrm{K}^{+}$-ATPase, inhibits bone resorption in vitro," Calcified Tissue International, vol. 38, no. 2, pp. 123-125, 1986.

[14] R. Gruber, "Molecular and cellular basis of bone resorption," Wiener Medizinische Wochenschrift, 2014.

[15] J. P. Mattsson, K. Väänänen, B. Wallmark, and P. Lorentzon, "Omeprazole and bafilomycin, two proton pump inhibitors: differentiation of their effects on gastric, kidney and bone $\mathrm{H}^{+}$translocating ATPases," Biochimica et Biophysica Acta: Biomembranes, vol. 1065, no. 2, pp. 261-268, 1991.

[16] K. B. Filion, D. Chateau, L. E. Targownik et al., "Proton pump inhibitors and the risk of hospitalisation for communityacquired pneumonia: replicated cohort studies with metaanalysis," Gut, vol. 63, pp. 552-558, 2014.

[17] K. A. Yearsley, L. J. Gilby, A. V. Ramadas, E. M. Kubiak, D. L. Fone, and M. C. Allison, "Proton pump inhibitor therapy is a risk factor for Clostridium difficile-associated diarrhoea," Alimentary Pharmacology and Therapeutics, vol. 24, no. 4, pp. 613-619, 2006.

[18] A. R. Sheraly, D. Lickorish, F. Sarraf, and J. E. Davies, "Use of gastrointestinal proton pump inhibitors to regulate osteoclastmediated resorption of calcium phosphate cements in vivo," Current Drug Delivery, vol. 6, no. 2, pp. 192-198, 2009.

[19] J. J. Hyun, H. J. Chun, B. Keum et al., "Effect of omeprazole on the expression of transcription factors in osteoclasts and osteoblasts," International Journal of Molecular Medicine, vol. 26, no. 6, pp. 877-883, 2010.
[20] M. Zaidi, "Modularity of osteoclast behaviour and "modelspecific" inhibition of osteoclast function," Bioscience Reports, vol. 10, no. 6, pp. 547-556, 1990.

[21] L. E. Targownik, L. M. Lix, C. J. Metge, H. J. Prior, S. Leung, and W. D. Leslie, "Use of proton pump inhibitors and risk of osteoporosis-related fractures," Canadian Medical Association Journal, vol. 179, no. 4, pp. 319-326, 2008.

[22] K. Mizunashi, Y. Furukawa, K. Katano, and K. Abe, "Effect of omeprazole, an inhibitor of $\mathrm{H}^{+}, \mathrm{K}^{+}$-ATPase, on bone resorption in humans," Calcified Tissue International, vol. 53, no. 1, pp. 2125, 1993.

[23] J. Costa-Rodrigues, S. Reis, S. Teixeira, S. Lopes, and M. H. Fernandes, "Dose-dependent inhibitory effects of proton pump inhibitors on human osteoclastic and osteoblastic cell activity," The FEBS Journal, vol. 280, no. 20, pp. 5052-5064, 2013. 


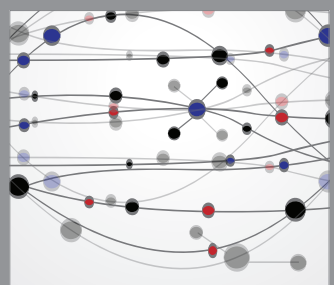

The Scientific World Journal
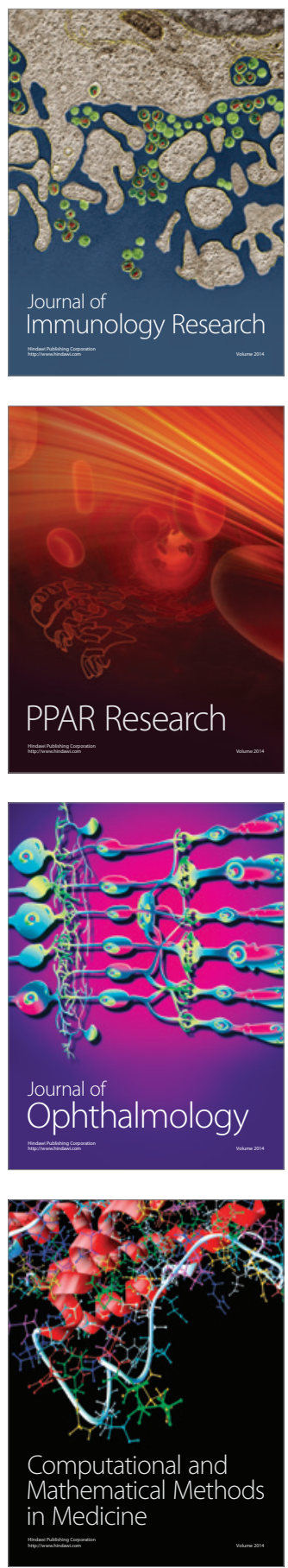

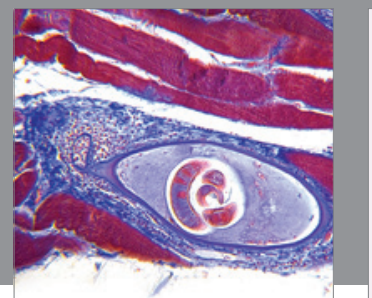

Gastroenterology

Research and Practice
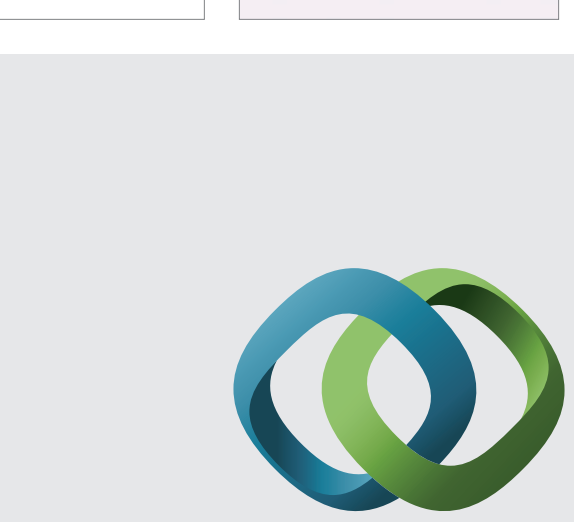

\section{Hindawi}

Submit your manuscripts at

http://www.hindawi.com
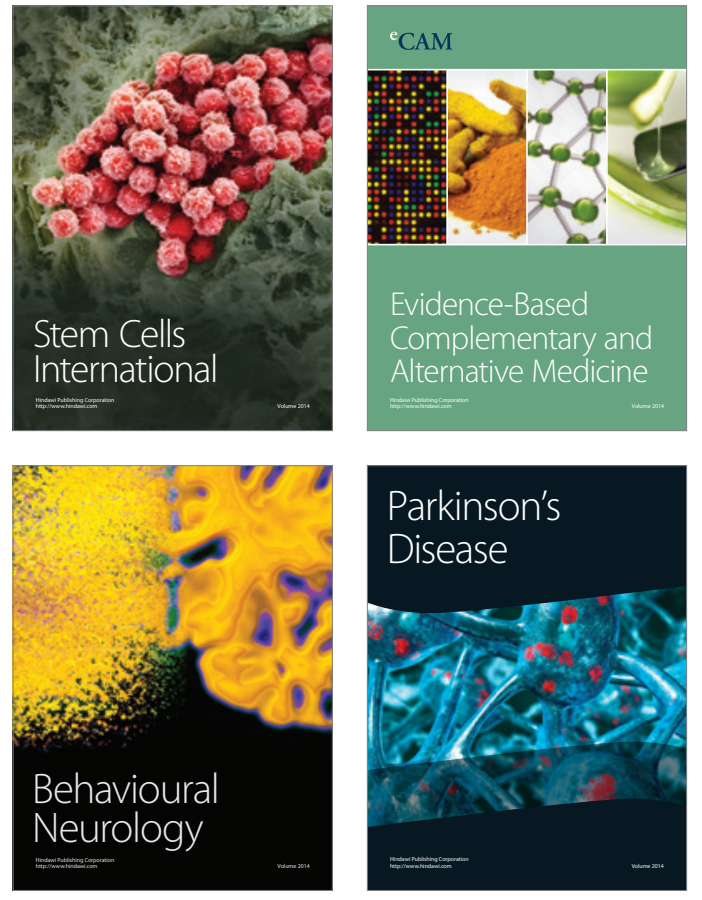
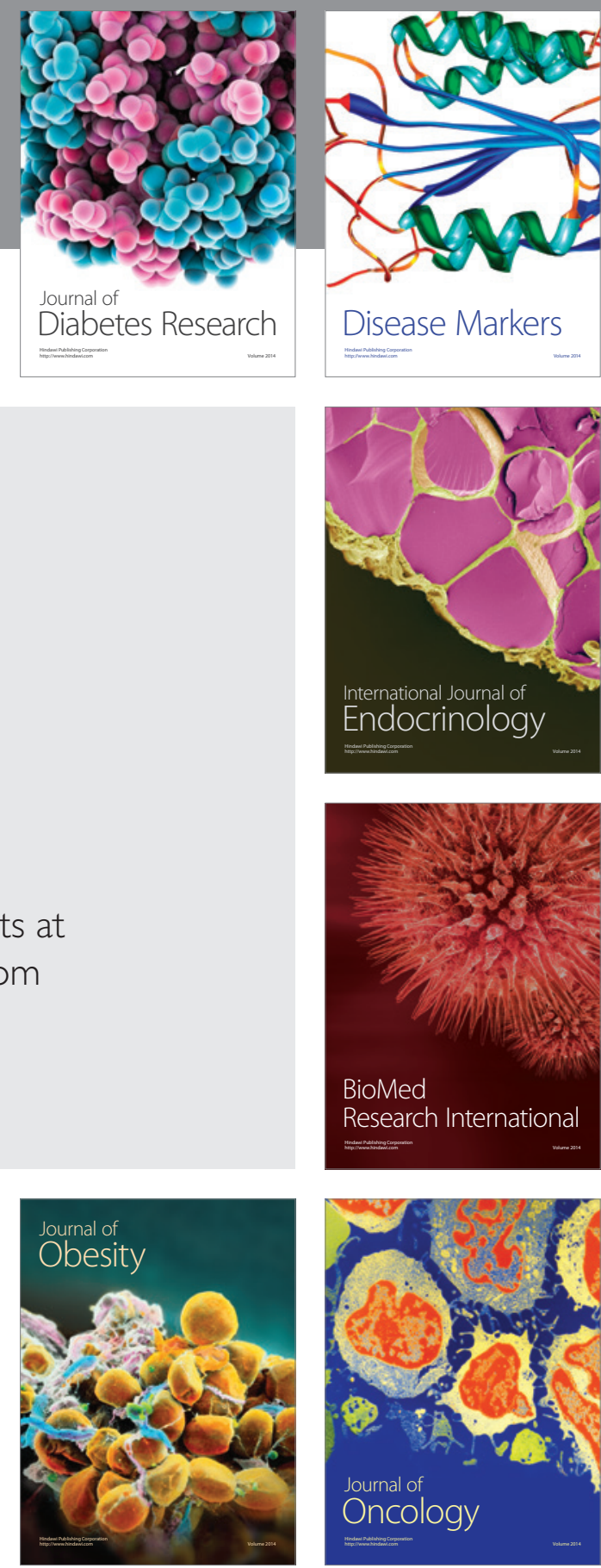

Disease Markers
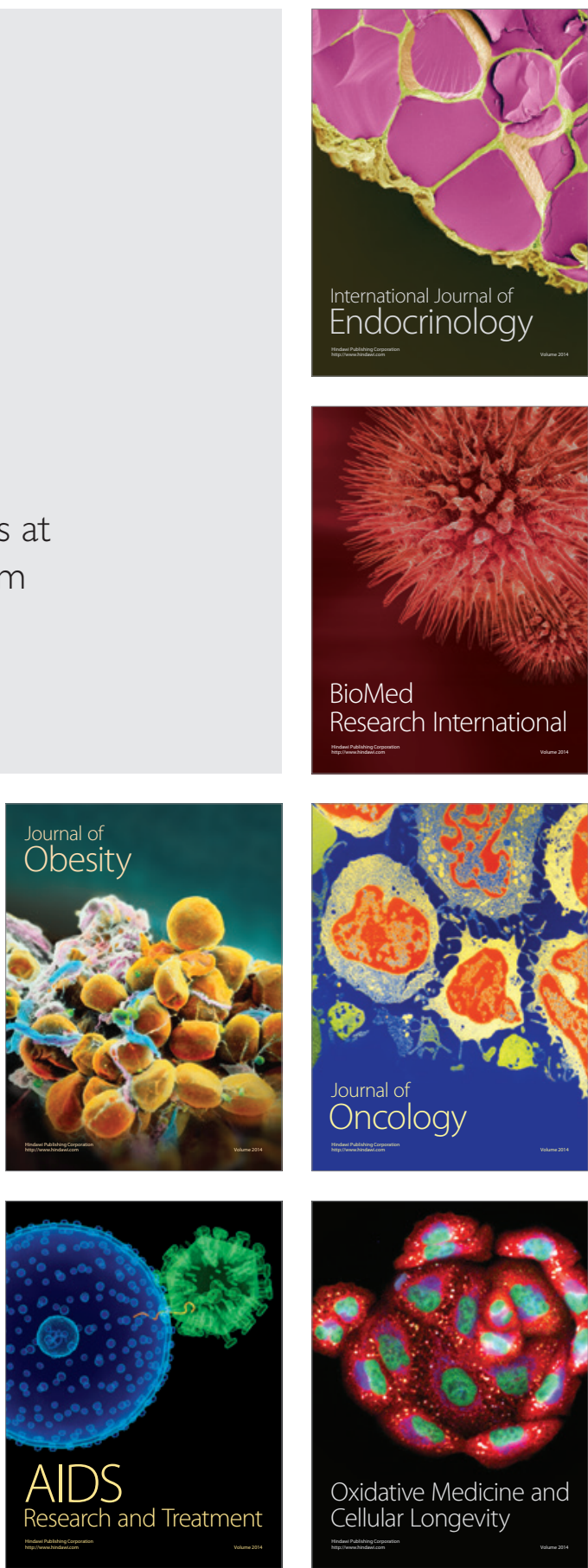